\title{
Molecular architecture of axonemal microtubule doublets revealed by cryo-electron tomography
}

\author{
Haixin Sui \& Kenneth H. Downing \\ Life Sciences Division, Lawrence Berkeley National Laboratory, Berkeley, CA 94720
}

The axoneme, which forms the core of eukaryotic flagella and cilia, is one of the largest macromolecular machines with a structure that is largely conserved from protists to mammals ${ }^{1}$. Microtubule doublets are structural components of axonemes containing a number of proteins besides tubulin, and are usually found in arrays of nine doublets arranged around two singlet microtubules. Coordinated sliding of adjacent doublets, which involves a host of other proteins in the axoneme, produces periodic beating movements of the axoneme. We have obtained a 3D density map of intact microtubule doublets using cryo-electron tomography and image averaging. Our map, with a resolution of about $3 \mathrm{~nm}$, provides insights into locations of particular proteins within the doublets and the structural features of the doublets that define their mechanical properties. We identify likely candidates for several of these non-tubulin components of the doublets. This work offers novel insight on how tubulin protofilaments and accessory proteins attach together to form the doublets and provides a structural basis for understanding doublet function in axonemes.

Microtubule doublets isolated from sea urchin sperm were studied by cryo-electron tomography. Groups of up to nine parallel doublets, apparently from a single axoneme, are often found in our frozen-hydrated samples, as shown in Figs. 1 and S2. The protofilaments (PFs) as well as the $4 \mathrm{~nm}$ tubulin monomer repeat along the PFs, are already resolved in the unaveraged tomographic reconstructions (Figs. 1 and 2a). As described in the methods section, small 3D volumes along each doublet were extracted from the tomograms and then aligned and averaged. Combining data from nine doublets produced an improved density map, as 
illustrated in Fig. 2b. Because of the limited angular range over which data is collected the map shows better separation between PF densities in the plane of the specimen than perpendicular to it.

Axonemes contain a number of proteins in addition to tubulin, generally with periodicities that are multiples of the $8-\mathrm{nm}$ tubulin dimer repeat. The longest periodicity detectable in our data from the isolated doublets was $16 \mathrm{~nm}$, so the final map was obtained by filtering the Fourier transform using the layer lines at multiples of $1 /(16 \mathrm{~nm})$. Figure $2 \mathrm{c}$ is the axial projection of the final density map and shows that the microtubule doublet consists of a complete singlet microtubule, the A-tubule containing $13 \mathrm{PFs}$, and an incomplete microtubule, the B-tubule, containing 10 PFs.

In order to interpret structural features of the doublets, we built a pseudo-atomic model of the tubulin component by docking the crystal structure of the $\alpha / \beta$ tubulin $\mathrm{PF}^{2}$ into the $3 \mathrm{D}$ density map (Fig. 3a). Unlike singlet microtubules which are circular in cross section (as in Fig. 1c), the A-tubules show a slight elliptical deformation with an elongation of about $8 \%$ in the axoneme's radial dimension. We introduced this distortion in a model of a 13-PF microtubule with a mean radius of $112 \AA$ to construct the A-tubule. The B-tubule was built of 10 straight PFs with a mean radius of $129 \AA$, which corresponds to the size of a 15-PF microtubule, with a similar distortion in PF positions. For convenience in further discussion, we number PFs as shown in Fig. 3a. To judge the quality of the docking we calculated a density from the atomic model by removing data from its Fourier transform corresponding to the missing data for the experimental map. This calculated density matched the experimental density very well (Fig. 3b), accounting for effects of the anisotropic resolution. The model density accounts for almost all of the experimental density, and the high degree of similarity between the two maps throughout most of the structure makes it straightforward to identify features that represent non-tubulin components of the doublet. A difference map (Figs. 3c-f) highlights densities that are clearly not accounted for by tubulin.

Toward the outer side of the axoneme, PF B10 is in close contact with A4 and A5 apparently making a tight connection between the A- and B-tubules. In the present model the 
B10-A5 contact actually contains steric clashes, but the density does not provide sufficient resolution to reliably modify the model and resolve the clashes. Toward the inner side of the axoneme, PF B1 makes closest approach to A1 but with a gap between the A- and B-tubules.

The region containing PFs A1-A4, referred to as the partition, covers the open part of the B-tubule and separates the hollow space of the two tubules. The PFs of this region can be isolated as a stable ribbon of three adjoining $\mathrm{PFs}^{3-6}$. Our map shows how some of the other proteins bound in the partition region help to stabilize this ribbon.

The most distinctive density inside the A-tubule is next to the PFs of the partition, similar to features previously observed in cross-sections of plastic embedded doublets ${ }^{7}$. This density is resolved as a filamentous structure running mainly along PF A3 with projections extending out across several adjacent PFs. Figure $3 \mathrm{~d}$ is a side view of this region showing three densities running across the partition. Two of these densities extend to PF A1, with an axial repeat of $8 \mathrm{~nm}$, and the other runs across PFs A4 and A5 with a periodicity of $16 \mathrm{~nm}$. These densities form a distinctive bridge-like structure in the longitudinal projection (Fig. 2c) which we term the partition bridge density.

The ribbon formed by the partition contains only a few proteins besides tubulin, including tektins $\mathrm{A}, \mathrm{B}$ and $\mathrm{C}^{6}$. In TEM studies, a filament which has been shown to contain tektin is frequently seen extending from the end of the ribbon ${ }^{8-10}$ (also Fig. S3). Thus one can surmise that the only continuous density in the tomogram associated with the ribbon PFs is tektin.

Tektins are predicted to share structural features with intermediate filament proteins $(\text { IFPs })^{8,10-15}$, forming heterodimers or homodimers with two globular heads and a coiled-coil structure extending in a tail. Crosslinking studies indicate that tektins A and B form a heterodimer ${ }^{16}$. The present density map is compatible with a number of models for how the tektins are arranged, but a likely interpretation is that the two domains pointing to the right in Fig. $3 \mathrm{~d}$ correspond to parts of tektin A/B heterodimers, while the density pointing to the left is 
part of a tektin $\mathrm{C}$ homodimer. The continuous filament would then be composed of the head domains, possibly along with parts of the helical domains.

Also on the inside of the A-tubule, there are distinctive densities contacting PFs A7 A13. The density interacting with A10 and A11 protrudes into the lumen of the A-tubule. Another density between PF A12 and A13 projects to the outside of the A-tubule (Fig. 3a-3c).

As shown in Fig. 3a, there is a small density projecting out from the doublet in the region of the outer junction between the A- and B-tubules. Because of the positioning of PF $\mathrm{B} 10$ in the present model, this feature does not appear in the difference density at the isosurface level used in Fig. 3c. However, if B10 is positioned to produce more realistic contacts with A4 and A5 one could interpret the density as being a small accessory protein that would stabilize the B10-A5 interaction. This protein could bind to both B10 and A5 in the region occupied by other MAPs, but its identity is still unclear.

Our 3D density map reveals a connection every $16 \mathrm{~nm}$ between the A and B-tubules toward the inner region (Figs. 3e), which we refer to as the linker density. The linker density connects PF A2 to B1 and extends to PF B2 on one side, with an extension that links PFs A2 and $\mathrm{A} 3$ on the outside of the A-tubule. The structure of this linker appears to provide a flexibility that would allow easier twisting of the doublet than if it were a rigid connection. There is another very different density along PF B1 between the linker densities. Some studies had suggested that there might be another PF of the B-tubule in this region making a direct contact with the A-tubule, but our reconstruction shows that this is not the case.

The most likely candidates for the molecular components of the linker are Sp77 and Sp83, which co-purify with the isolated ribbons ${ }^{6}$. Densities with a periodicity of $16 \mathrm{~nm}$ are observed at the edge of ribbons ${ }^{3,7}$ (see also Fig. S3), where immunolabeling studies have identified both $\mathrm{Sp} 77$ and $\mathrm{Sp} 83^{17}$, as well as Rib72, a Chlamydomonast homologue of Sp77 ${ }^{18,19}$. Immunofluorescence microscopy has also shown that an anti-Sp77 antibody labels the 9 doublets but not the central pair singlets, further supporting the hypothesis that $\mathrm{Sp} 77$ interacts with both the A- and B-tubules. 
The linker density in the tomogram appears to represent a protein significantly larger than $77 \mathrm{kDa}$ and thus probably contains other protein(s). Since Sp77 and Sp83 are present in essentially the same amount, it is likely that Sp83 also contributes to the linker protein. Sp83 was found in both doublets and the central pair ${ }^{17}$, suggesting that its binding may be more similar to a conventional MAP on the A-tubule rather than directly linking to the B-tubule.

Within the axoneme, there are highly dynamic interactions between doublets arising from the inner and outer dyneins, as well as stable connections formed by nexin ${ }^{20}$. Nexin has been localized in previous EM studies near the inner side of the doublets ${ }^{21,22}$. In some of our tomograms where the axonemes are less disrupted, we see a filamentous density that we interpret as nexin connecting PF B2 from one doublet to PFs A9-A11 on the adjacent doublet (Fig. S2). Rib72 is also implicated in forming the inter-doublet linkages ${ }^{19}$ and may do so via interactions with nexin near PF B2.

Our interpretation of the arrangement of proteins found in the microtubule doublets along with associated proteins described elsewhere in the literature is summarized in Fig. S1 . The proteins in the partition bridge density, together with the linker density attached to the partition wall outside the A-tubule, clearly play a critical role in stabilizing the PFs near the partition. It appears that they may account for the elliptical distortion of the A-tubule, and they may also provide a substantial increase in resistance to bending of the doublet out of the plane. Other proteins of the A-tubule are located in regions where they could reinforce the tube and help to anchor proteins that bind on the outside. For example, there are distinctive densities inside the regions where the dynein heads are located. The largest feature, on PFs A10-A12, sits inside the attachment region for nexin and the dynein regulatory complex with which it is associated $^{23-26}$. The density we observe between PFs 12 and 13 is at the point where the radial spokes meet the doublets and may be part of their attachment. These external proteins have larger periodicities than the features we see in the doublets, and further work will be required to fully understand the significance of their interactions. The proteins that bind inside the A-tubule do so in a way very different from other MAPs, with the possible exception of $\operatorname{tau}^{27}$, linking adjacent PFs in ways that seem to supplement the normal inter-PF interactions. 
The configuration of the proteins that comprise the microtubule doublet appears to be designed to stabilize and maintain the protofilament architecture of the doublet as it undergoes the stresses involved in axoneme motion and also to favor bending in the direction that corresponds to twisting of the axoneme. Our structure of the microtubule doublet provides insights into several novel tubulin-protein interactions and the function doublets performs in axonemes, and will serve as a greatly improved basis for quantitative modeling of mechanical properties.

\section{METHODS}

Sperm flagella axonemes were prepared from the sea urchin, Strongylocentrotus purpuratus, following the protocol in ref ${ }^{28}$. For the PF ribbon samples, material was treated with $0.7 \%$ Sarkosyl in $10 \mathrm{mM}$ Tris- $\mathrm{HCl}(\mathrm{pH}: 7.8)$ at $4 \mathrm{C}$ for about 6 hours and then centrifuged at $100,000 \mathrm{~g}$ for 30 minutes. The pellet was collected and resuspended in the above Tris buffer without Sarkosyl.

The concentrated doublet sample was resuspended in water containing 5 or $10 \mathrm{~nm}$ colloidal gold particles (Ted Pella, Inc), and applied to a grid covered with a glow-discharged holey carbon film. Grids were blotted, plunge-frozen in liquid ethane and transferred under liquid nitrogen to the cryo-tilt holder of a JEOL 3100FEF electron microscope. The microscope, operating at $300 \mathrm{kV}$, was equipped with an Omega energy filter set to a slit width of $25 \mathrm{eV}$. Microtubule doublets nearly parallel to the tilt axis were selected for data collection. Tomographic data were collected under low-dose condition with accumulated dose about 6500 electrons per $\mathrm{nm}^{2}$. Single-axis tilt series included $33-46$ images with a tilt increment of $3-4$ degrees, were recorded at a defocus of 3.5 to $5 \mu \mathrm{m}$ on a $2048 \times 2048$ CCD (Gatan, Inc). The final pixel size of the images was about $0.53 \mathrm{~nm}$.

3D segment volumes along each doublet microtubule were extracted from the tomographic reconstructions. The volumes were aligned and averaged as described in the Supplementary Materials. 
For modeling, the atom coordinates of the PF models for the A- and B-tubules were separately calculated as described in the text using the backbone atoms from the tubulin crystal structure, PDB ID 1JFF, and manually shifted into the density as a whole.

\section{References}

1. Kohl, L. \& Bastin, P. The flagellum of trypanosomes. Int Rev Cytol 244, 227-85 (2005).

2. Nogales, E., Wolf, S. G. \& Downing, K. H. Structure of the alpha beta tubulin dimer by electron crystallography. Nature 391, 199-203 (1998).

3. Witman, G. B., Carlson, K., Berliner, J. \& Rosenbaum, J. L. Chlamydomonas flagella. I. Isolation and electrophoretic analysis of microtubules, matrix, membranes, and mastigonemes. J Cell Biol 54, 507-39 (1972).

4. Witman, G. B., Carlson, K. \& Rosenbaum, J. L. Chlamydomonas flagella. II. The distribution of tubulins 1 and 2 in the outer doublet microtubules. J Cell Biol 54, 540-55 (1972).

5. Meza, I., Huang, B. \& Bryan, J. Chemical heterogeneity of protofilaments forming the outer doublets from sea urchin flagella. Exp Cell Res 74, 535-40 (1972).

6. Linck, R. W. \& Norrander, J. M. Protofilament ribbon compartments of ciliary and flagellar microtubules. Protist 154, 299-311 (2003).

7. Linck, R. W. Flagellar doublet microtubules: fractionation of minor components and alpha-tubulin from specific regions of the A-tubule. J Cell Sci 20, 405-39 (1976).

8. Linck, R. W. \& Langevin, G. L. Structure and chemical composition of insoluble filamentous components of sperm flagellar microtubules. J Cell Sci 58, 1-22 (1982).

9. Linck, R. W., Amos, L. A. \& Amos, W. B. Localization of tektin filaments in microtubules of sea urchin sperm flagella by immunoelectron microscopy. J Cell Biol 100, 126-35 (1985).

10. Linck, R. W. \& Stephens, R. E. Biochemical characterization of tektins from sperm flagellar doublet microtubules. J Cell Biol 104, 1069-75 (1987). 
11. Chang, X. J. \& Piperno, G. Cross-reactivity of antibodies specific for flagellar tektin and intermediate filament subunits. J Cell Biol 104, 1563-8 (1987).

12. Linck, R. W., Albertini, D. F., Kenney, D. M. \& Langevin, G. L. Tektin filaments: chemically unique filaments of sperm flagellar microtubules. Cell Motility Supplement 1, 127-32 (1982).

13. Chen, R., Perrone, C. A., Amos, L. A. \& Linck, R. W. Tektin B1 from ciliary microtubules: primary structure as deduced from the cDNA sequence and comparison with tektin A1. J Cell Sci 106 ( Pt 3), 909-18 (1993).

14. Norrander, J. M., Amos, L. A. \& Linck, R. W. Primary structure of tektin A1: comparison with intermediate-filament proteins and a model for its association with tubulin. Proc Natl Acad Sci U S A 89, 8567-71 (1992).

15. Steffen, W. \& Linck, R. W. Relationship between tektins and intermediate filament proteins: an immunological study. Cell Motil Cytoskeleton 14, 359-71 (1989).

16. Pirner, M. A. \& Linck, R. W. Tektins are heterodimeric polymers in flagellar microtubules with axial periodicities matching the tubulin lattice. J Biol Chem 269, 31800-6 (1994).

17. Hinchcliffe, E. H. \& Linck, R. W. Two proteins isolated from sea urchin sperm flagella: structural components common to the stable microtubules of axonemes and centrioles. J Cell Sci 111 ( Pt 5), 585-95 (1998).

18. Patel-King, R. S., Benashski, S. E. \& King, S. M. A bipartite Ca2+-regulated nucleoside-diphosphate kinase system within the Chlamydomonas flagellum. The regulatory subunit p72. J Biol Chem 277, 34271-9 (2002).

19. Ikeda, K. et al. Rib72, a conserved protein associated with the ribbon compartment of flagellar A-microtubules and potentially involved in the linkage between outer doublet microtubules. J Biol Chem 278, 7725-34 (2003).

20. Lindemann, C. B. Testing the geometric clutch hypothesis. Biol Cell 96, 681-90 (2004).

21. Bozkurt, H. H. \& Woolley, D. M. Morphology of nexin links in relation to interdoublet sliding in the sperm flagellum. Cell Motil Cytoskeleton 24, 109-18 (1993).

22. Woolley, D. M. Studies on the eel sperm flagellum. I. The structure of the inner dynein arm complex. J Cell Sci 110 ( Pt 1), 85-94 (1997). 
23. Huang, B., Ramanis, Z. \& Luck, D. J. Suppressor mutations in Chlamydomonas reveal a regulatory mechanism for flagellar function. Cell 28, 115-24 (1982).

24. Gardner, L. C., O'Toole, E., Perrone, C. A., Giddings, T. \& Porter, M. E. Components of a "dynein regulatory complex" are located at the junction between the radial spokes and the dynein arms in Chlamydomonas flagella. J Cell Biol 127, 1311-25 (1994).

25. Piperno, G., Mead, K., LeDizet, M. \& Moscatelli, A. Mutations in the "dynein regulatory complex" alter the ATP-insensitive binding sites for inner arm dyneins in Chlamydomonas axonemes. J Cell Biol 125, 1109-17 (1994).

26. Piperno, G., Mead, K. \& Shestak, W. The inner dynein arms I2 interact with a "dynein regulatory complex" in Chlamydomonas flagella. J Cell Biol 118, 1455-63 (1992).

27. Kar, S., Fan, J., Smith, M. J., Goedert, M. \& Amos, L. A. Repeat motifs of tau bind to the insides of microtubules in the absence of taxol. Embo J 22, 70-7 (2003).

28. Waterman-Storer, C. M. Microtubule/organelle motility assays. Current Protocols in Cell Biology, Bonifacino, J.S., Dasso, M., Harford, J.B., Lippincott-Schwartz, J., and Yamada, K.M. eds. John Wiley, NY Unit 13.1, 13.1.6-13.1.7 (1998).

29. Hoops, H. J. \& Witman, G. B. Outer doublet heterogeneity reveals structural polarity related to beat direction in Chlamydomonas flagella. J Cell Biol 97, 902-8 (1983).

30. Mastronarde, D. N., O'Toole, E. T., McDonald, K. L., McIntosh, J. R. \& Porter, M. E. Arrangement of inner dynein arms in wild-type and mutant flagella of Chlamydomonas. J Cell Biol 118, 1145-62 (1992).

Supplementary Information is linked to the online version of the paper at www.nature.com/nature

Acknowledgements We thank Alison Killilea for sea urchin collection, Keith Gull for discussions on protofilament numbering, Huilin Li, Beata Rockel and Dieter Typke for discussion and help on image processing, Bernard Heymann and Chunying Yang on BSOFT library usage, and Robert Glaeser and Manfred Auer for valuable suggestion and 
encouragement. This work is supported by NIH grants GM46033 and GM51487 and by the U.S. Department of Energy under Contract No. DE-AC02-05CH11231.

Author Information Reprints and permission information is available at npg.nature.com/reprintsandpermissions. The authors declare no competing financial interests. Correspondence and requests for materials should be addressed to K.H.D. (khdowning@lbl.gov).

\section{Figure Legends}

Figure 1. Electron tomography of microtubule doublets. (a) Doublets from a single axoneme are often found parallel to each other as seen in the zero-tilt image from one tomographic series. (b) Protofilaments are well resolved in the reconstructions, as shown in a $1 \mathrm{~nm}$-thick section of this tomogram roughly parallel to the doublets. (c) A projection of a $26 \mathrm{~nm}$-thick cross-section at the location indicated by line in Fig $1 \mathrm{~b}$. The field includes 5 complete and 2 broken doublets (black arrows indicate missing PFs) as well as two singlet microtubules, one of which has partially disassembled (white arrow).

Figure 2. Results of averaging tomographic data. (a) Power spectrum of the projection of one doublet from the tomographic reconstruction. The doublet image was computationally straightened before computing the Fourier transform. The layer-line at $4 \mathrm{~nm}$ is the most prominent. (b) Power spectrum of the averaged tomogram from 9 doublets, showing prominent layer-lines at multiples of $1 /(16 \mathrm{~nm})$ and extending to a resolution better than $3 \mathrm{~nm}$. (c) Projection image of the final doublet density map along the longitudinal axis. The orientation was determined by comparison with other work in which doublets have been visualized in the context of the axoneme $\mathrm{e}^{29,30}$. The view is from the proximal end of the axoneme, and the upward direction corresponds to the outer direction of the axoneme.

Figure 3. Interpretation of the 3D density map. (a) Axial view, seen from the proximal end, of the tomographic reconstruction that was filtered to enhance 16-nm spacings, viewed as an 
isosurface (gold). The pseudo-atomic model (backbone only) fit to the doublet density is shown in blue with labels assigned to the protofilaments. (b) Projection view of the experimental density in gold and density calculated from the pseudo-atomic model in purple.(c) Same views as above with difference map shown in green and model PF density in purple. (d, e, f) Side views of the difference map (green) and model density in purple. The orientations are indicated by the red lines in (c).

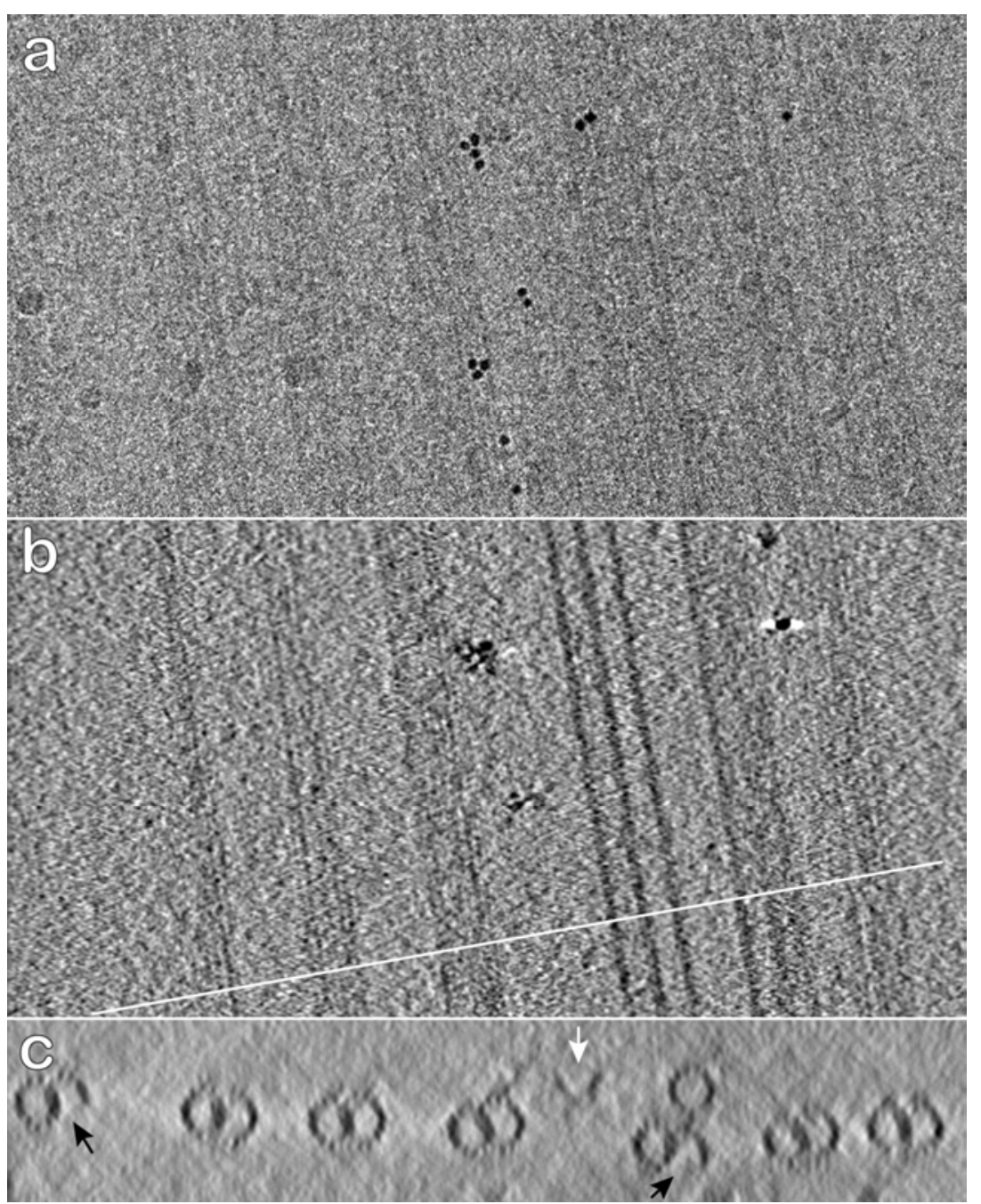

Fig 1 


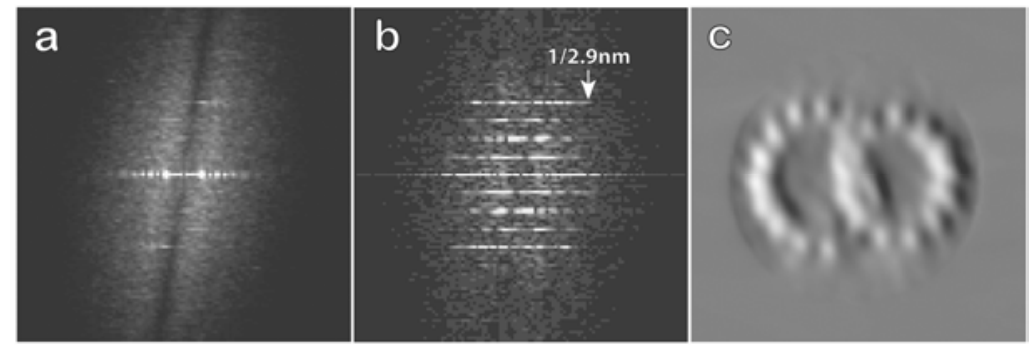

Fig. 2
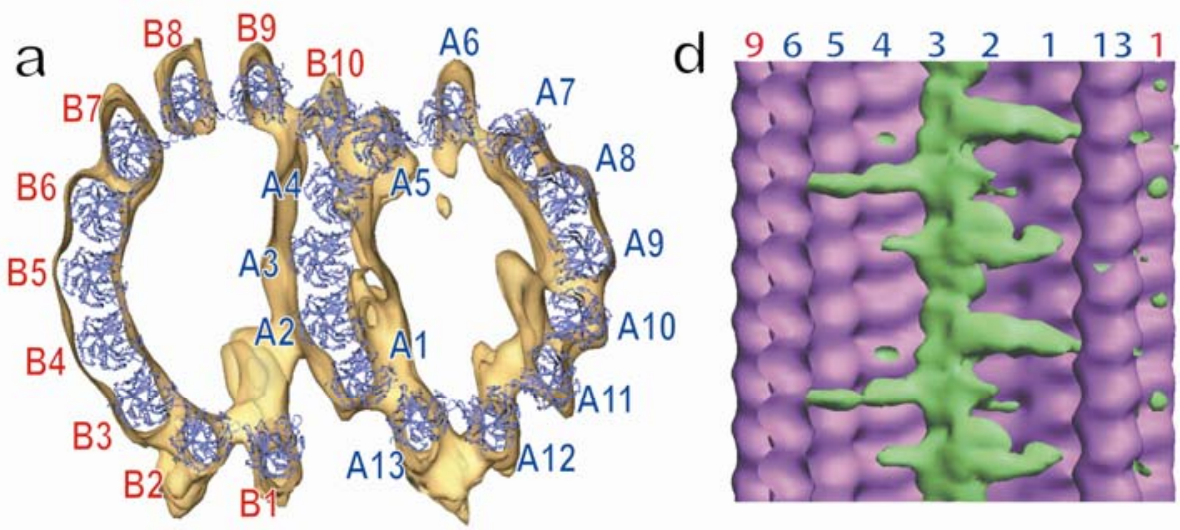

b
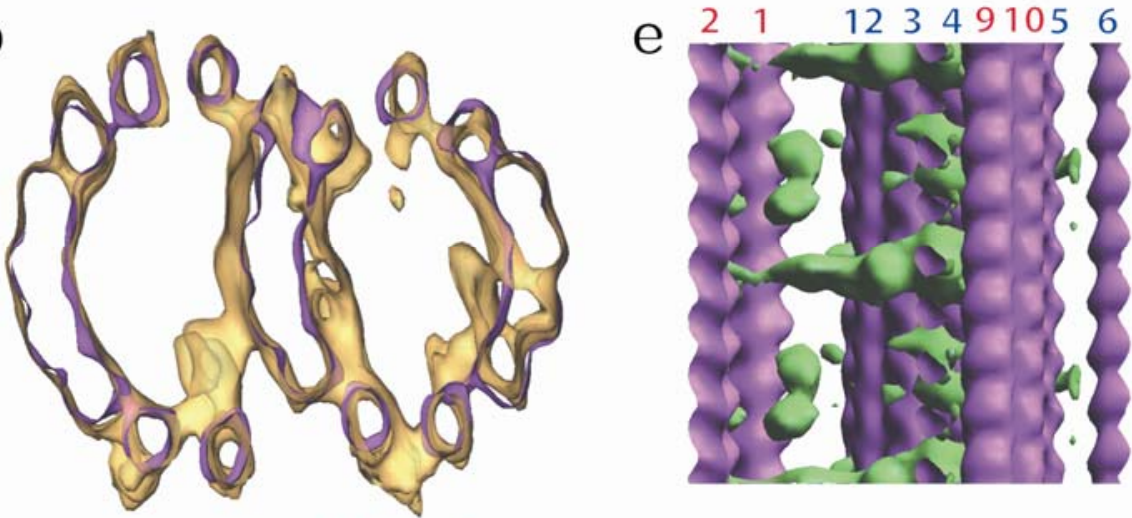

C
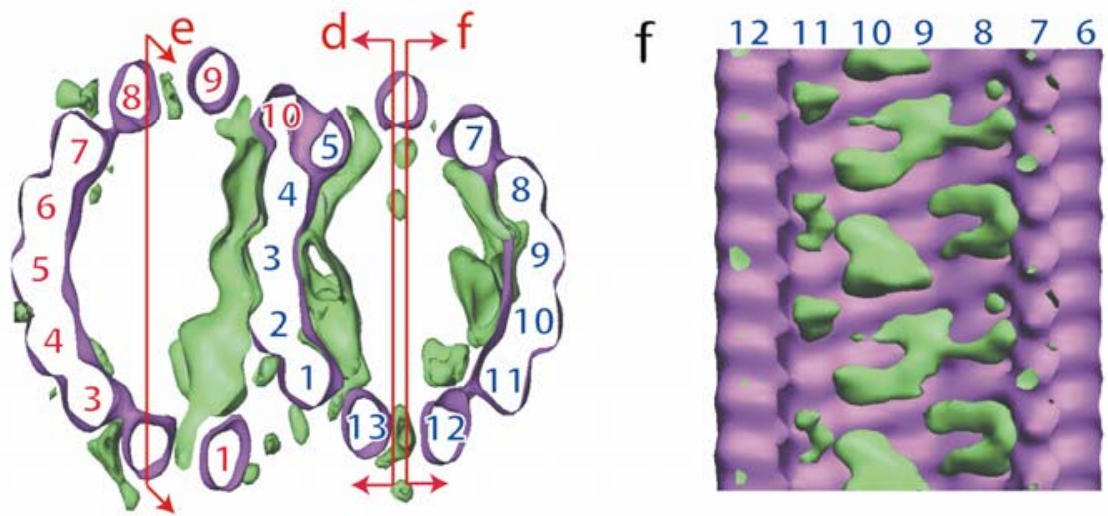

Fig. 3 


\section{Supplementary Materials}

Microtubule doublets isolated from sea urchin sperm were studied by cryoelectron tomography, producing a density map in which we can identify a number of nontubulin components, in addition to 23 tubulin protofilaments (PFs) that make up the Aand B-tubules. Figure S1 indicates the arrangements of these components and our interpretation of the identity of several of the non-tubulin proteins.

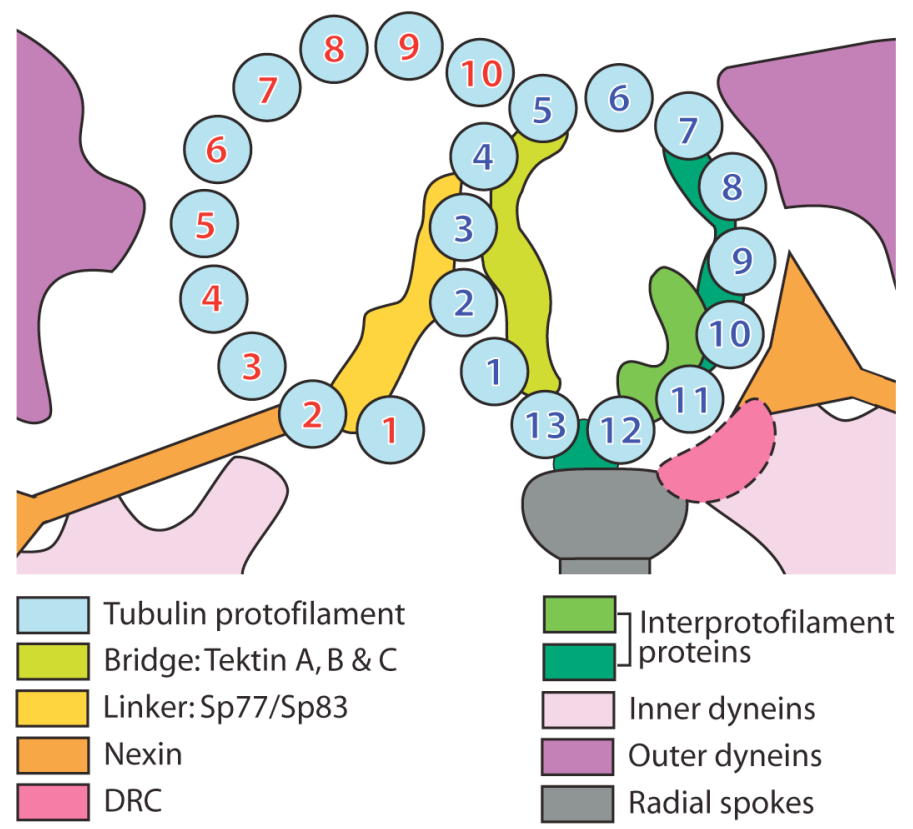

Figure S1. Schematic of proteins associated with microtubule doublets. Locations of tektins, Sp77, Sp83 and nexin are from this work; locations and shapes of dyneins and spokes $^{31,32}$ and the dynein regulatory complex ${ }^{33}$ are from previous publications. 

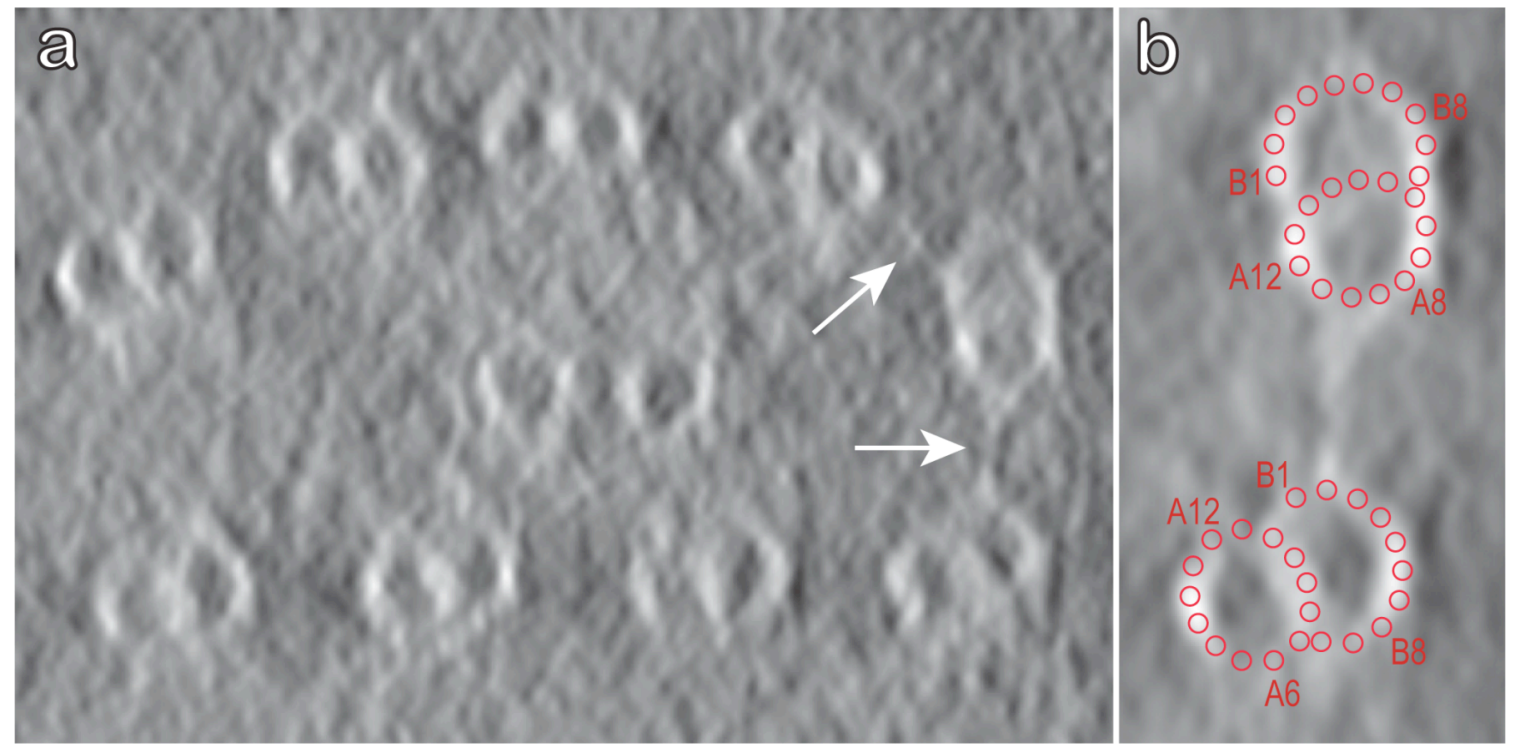

Figure S2 Electron tomogram of a region containing associated doublets. Because this sample was thicker than others, the resolution was not as good as in those used for the doublet map, but some of the inter-doublet linkages are still present. (a) Projection view of a $42 \mathrm{~nm}$-thick cross-section of a denoised cryo-tomogram (20 cycles of anisotropic diffusion). Around the central pair singlets, 9 doublets are parallel to each other. Densities can be seen linking several pairs of the doublets (arrows). (b) Projection images of seven $21 \mathrm{~nm}$-thick cross-sections, extracted from the tomogram shown in (a), were averaged to enhance the linkage density that is interpreted as nexin. Red circles indicate PF positions and indicate that the linker connects PF B2 to the region of A9-A11. Several of the PFs are labeled for reference. 


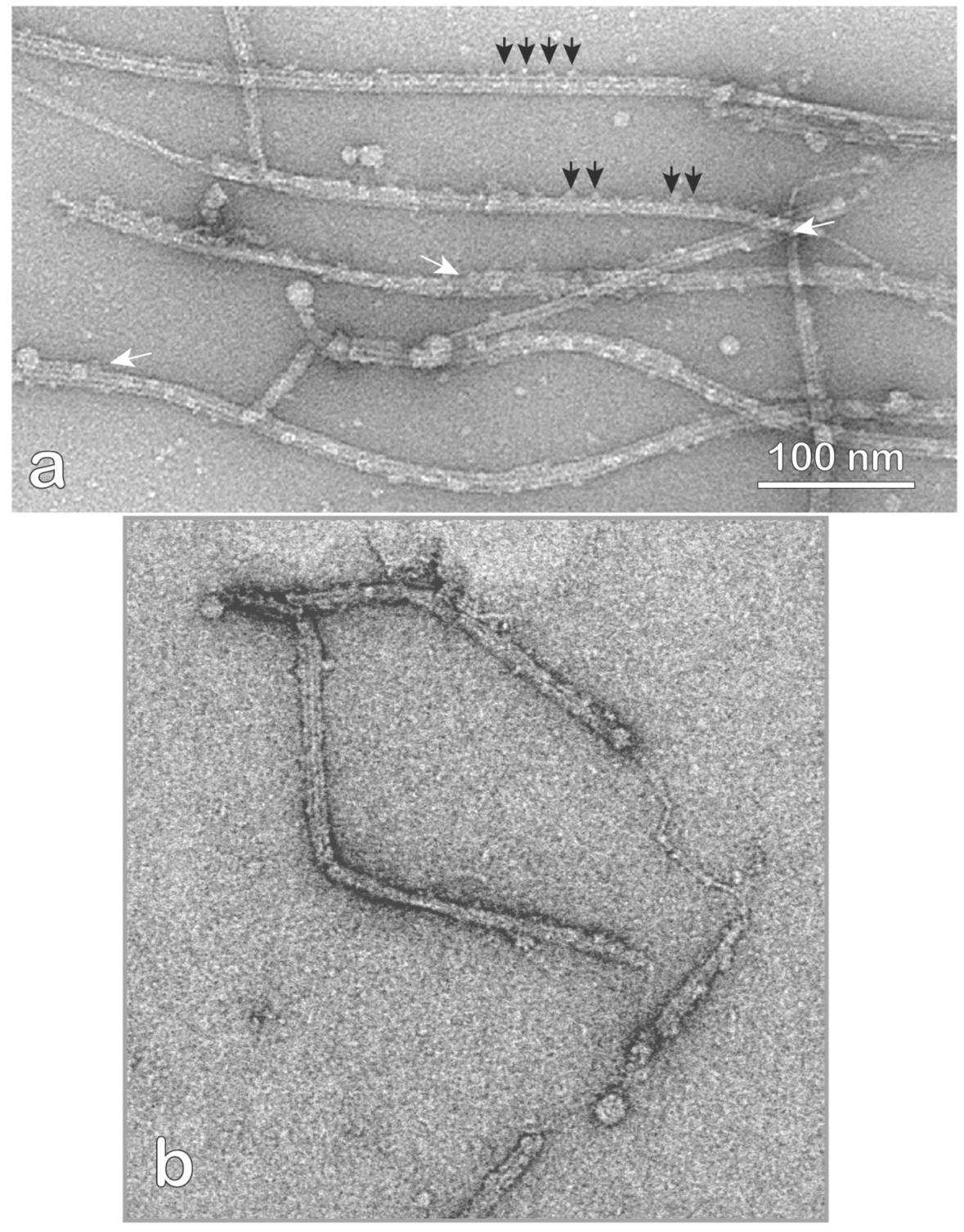

Figure S3 TEM micrograph of negatively stained ribbons (a) shows that the ribbons are formed mostly by 3 protofilaments with a fourth protofilament occasionally present (white arrows). Black arrows point to some of the densities spaced $16 \mathrm{~nm}$ apart along the edge of ribbons. (b) Area that shows single fibers extending from ends of some ribbons. 


\section{Supplementary Methods}

\section{Sample preparation and TEM data collection}

Sperm flagella axonemes were prepared from the sea urchin, Strongylocentrotus purpuratus, following the protocol in ref 34 . In brief, $0.55 \mathrm{M} \mathrm{KCl}$ was injected into the body cavity to introduce spawning. Membranes were osmotically removed using 20\% sucrose. Sperm heads were broken from the tails with a Dounce glass homogenizer and removed by centrifugation at $12,000 \mathrm{~g}$. After extensive homogenization and high salt washes $(0.6 \mathrm{M} \mathrm{NaCl})$, doublet microtubules were ready for further study. For the PF ribbon samples, the above material was treated with $0.7 \%$ Sarkosyl in $10 \mathrm{mM}$ Tris- $\mathrm{HCl}$ $(\mathrm{pH} \mathrm{7.8)}$ at $4 \mathrm{C}$ for about 6 hours and then centrifuged at 100,000g for 30 minutes. The pellet was collected and resuspended in the above Tris buffer without Sarkosyl.

The concentrated doublet sample was resuspended in water containing 5 or $10 \mathrm{~nm}$ colloidal gold particles (Ted Pella, Inc), and applied to a grid covered with a previously glow-discharged holey carbon film. The grid was plunge-frozen in liquid ethane after briefly blotting with filter paper at the edge of the grid. Grids were transferred under liquid nitrogen to the cryo-tilt holder of a JEOL 3100FEF electron microscope operated at an acceleration voltage of $300 \mathrm{kV}$. The microscope was equipped with an Omega energy filter that was operated in the zero-energy-loss mode with a slit width of $25 \mathrm{eV}$. Microtubule doublets nearly parallel to the tilt axis were selected for data collection. Tomographic data were collected under low-dose condition with accumulated dose about 6500 electrons per $\mathrm{nm}^{2}$. Single-axis tilt series included $33-46$ images with a tilt increment of 3-4 degrees. Images were recorded with defocus of 3.5 to $5 \mu \mathrm{m}$ on a 2048x2048 CCD (Gatan, Inc). The final pixel size of the images was about $0.53 \mathrm{~nm}$.

The ribbon sample was applied to TEM grids covered with continuous carbon film for one minute and then stained with 3\% uranyl acetate for 1 minute before blotting. The air dried specimens were examined using a Zeiss EM10 microscope. 


\section{Tomographic reconstruction}

Using colloidal gold particles as fiducial markers, the 2D projection images were aligned with the IMOD software package ${ }^{35}$. The tomographic reconstructions were calculated by weighted back-projection. Within a tomogram, there were normally several doublet microtubules running almost parallel to each other.

\section{$3 D$ image processing and averaging}

$3 \mathrm{D}$ segment volumes of $128 \times 128 \times 192$ voxels were extracted from the cryotomograms along each doublet microtubule. After denoising by bilateral filtering ${ }^{36}$, the images were aligned by an orientation search in a limited angular range using crosscorrelation programs we developed with the BSOFT package library ${ }^{37}$. The initial orientation parameters were used to re-extract volumes with better orientation from the original tomograms in order to avoid resolution loss from interpolation. The extracted volumes were orientated with the axis of the doublet roughly parallel to the $\mathrm{z}$ axis and the missing wedge roughly 45 degrees away from the $\mathrm{x}$ and $\mathrm{y}$ axes.

To further improve the alignment, each individual 3D volume was then projected along the $\mathrm{x}$ axis onto a $2 \mathrm{D}$ image. The $\mathrm{PF}$ structure contributes strongly to the equator in the power spectrum of this projection, and a Radon transform of the power spectrum shows a strong peak indicating the rotational offset required to align the PF precisely parallel to the $\mathrm{z}$ axis in the projection ${ }^{38}$. The volume was then projected along the $\mathrm{y}$ axis for determination of the other rotation angle. Combining the above two rotation angles, the doublet $3 \mathrm{D}$ volumes were aligned parallel to the $\mathrm{z}$ axis.

The projection of the aligned $3 \mathrm{D}$ volume along the $\mathrm{z}$ axis showed a distinctive $\mathrm{PF}$ distribution and was used to calculate the rotational alignment around the $\mathrm{z}$ axis. After an orientation alignment by combining the above three rotation angles, cross-correlation was used to find the translational shifts. The parameters were used to re-extract 3D images along the doublet microtubule tomogram. 3D volumes were then averaged for each doublet microtubule. 
This procedure, developed using the SPIDER package ${ }^{39}$, was repeated a number of times for each doublet microtubule. The averaged volumes were aligned and averaged before layer line filtering in Fourier space was applied for the final density map.

\section{Visualization and Model building}

Surface and volume rendering were performed using Chimera ${ }^{40}$ and the Amira 3D visualization package (Mercury Computer Systems, San Diego). For the averaged density maps, no denoising or feature enhancement was applied.

For modeling, the atom coordinates of the PF models for the A- and B-tubules were separately calculated as described in the text using the backbone atoms from the tubulin crystal structure, PDB ID 1JFF, and manually shifted into the density as a whole using AmiraMol.

\section{Supplementary Literature Citations}

31. Mitchell, D. R. Chlamydomonas Flagella. J Phycology 36, 261-273 (2000).

32. Inaba, K. Molecular architecture of the sperm flagella: molecules for motility and signaling. Zoolog Sci 20, 1043-56 (2003).

33. Gardner, L. C., O'Toole, E., Perrone, C. A., Giddings, T. \& Porter, M. E. Components of a "dynein regulatory complex" are located at the junction between the radial spokes and the dynein arms in Chlamydomonas flagella. J Cell Biol 127, 1311-25 (1994).

34. Waterman-Storer, C. M. Microtubule/organelle motility assays. Current Protocols in Cell Biology, Bonifacino, J.S., Dasso, M., Harford, J.B., LippincottSchwartz, J., and Yamada, K.M. eds. John Wiley, NY Unit 13.1, 13.1.6-13.1.7 (1998).

35. Kremer, J. R., Mastronarde, D. N. \& McIntosh, J. R. Computer visualization of three-dimensional image data using IMOD. J Struct Biol 116, 71-6 (1996).

36. Jiang, W., Baker, M. L., Wu, Q., Bajaj, C. \& Chiu, W. Applications of a bilateral denoising filter in biological electron microscopy. J Struct Biol 144, 114-22 (2003).

37. Heymann, J. B. Bsoft: image and molecular processing in electron microscopy. J Struct Biol 133, 156-69 (2001).

38. Li, H., DeRosier, D. J., Nicholson, W. V., Nogales, E. \& Downing, K. H. Microtubule structure at 8 A resolution. Structure (Camb) 10, 1317-28 (2002).

39. Frank, J. et al. SPIDER and WEB: processing and visualization of images in 3D electron microscopy and related fields. J Struct Biol 116, 190-9 (1996). 
40. Pettersen, E. F. et al. UCSF Chimera--a visualization system for exploratory research and analysis. J Comput Chem 25, 1605-12 (2004). 